\title{
Cryptococcal meningoencephalitis leading to Binocular visual loss and paraparesis in a patient with advanced HIV
}

\author{
Authors
}

\section{Akhil Deshmukh', Amod Rajendran², Aravind Reghukumar ${ }^{3 *}$, Athul Gurudas ${ }^{4}$, Kiran Kumar ${ }^{5}$}

1,2,4,5 Department of Internal medicine, Medical College, Thiruvananthapuram, Kerala, India

${ }^{3} \mathrm{Head}$ of the Department of Infectious Diseases, Government Medical College, Thiruvananthapuram,

Kerala, India

*Corresponding Author

Aravind Reghukumar

Head of the Department of Infectious Diseases, Government Medical College, Thiruvananthapuram,

Kerala, India

Abstract
Cryptococcus neoformans is the most common invasive fungal infection of the central nervous system in
patients with advanced HIV. Neurocryptococcosis usually presents as diffuse meningoencephalitis with
ocular involvement occurring as a secondary phenomenon is up to $30 \%$ of cases. Binocular visual loss
due to cryptococcal meningoencephalitis being very rare, this case report describes a patient with
advanced HIV who developed binocular visual loss and paraparesis as a complication of
cryptococcaemia. Even though mechanisms of visual complications in cryptococcal meningitis are still
unclear, intracranial hypertension is postulated to be the primary cause while leads to delayed onset of
visual loss.
Our patient had early onset of binocular painless visual loss in the absence of papilloedema Patient also
had features of cryptococcal spinal arachnoiditis which manifested as asymmetric lower limb weakness
with urinary retention. In view of binocular visual loss and spinal arachnoiditis, adjunctive
corticosteroids were added to the treatment regime consisting of amphotericin deoxycholate and
flucytosine, following which the patient made a gradual clinical recovery.
Keywords: Cryptococcal meningitis; Spinal arachnoiditis; Visual loss; Cryptococcoma; HIV.

\section{Introduction}

Cryptococcus neoformans is an encapsulated basidiomycete yeast which is usually found in decaying organic material and pigeon excreta ${ }^{(1)}$. Cryptococcus is a saprobe in nature, associated with certain trees (Eucalyptus) and rooting wood, soil contaminated by bird droppings. Cryptococcus family includes two pathogenic species; cryptococcus neoformans and cryptococcus gatti which grow at temperature 30- 40deg C (37deg C). The two most common sites for infection are the lung and central nervous system. Other sites being skin, prostate, and eye. The respiratory tract is the most common portal of entry for this yeast and symptoms range from asymptomatic colonization to life-threatening pneumonia with evidence of acute respiratory distress syndrome. Immunosuppressed patients have extrapulmonary more commonly than pulmonary manifestations. 
The central nervous system is the most common site in immunosuppressed individuals ${ }^{(2)}$. The organism has selective neurotropism due to enzyme laccase which converts phenolic compounds like norepinephrine and dopamine to melanin ${ }^{(3)}$. Melanin and polysaccharide capsule helps in pathogenicity of fungi ${ }^{(4)}$. The use of India ink staining remains a common diagnostic tool for identifying Cryptococcus in cerebrospinal fluid $(\mathrm{CSF})^{(5)}$, yet the sensitivity of India ink microscopy is only $<86 \%{ }^{(6)}$.

Culture is known to be the gold standard for the diagnosis of cryptococcal meningitis but has some drawbacks $^{(7)}$. Fungal cultures require laboratory facilities, electrical equipment, and qualified technicians. Cultures can take up to 7 days to develop and need to be incubated for up to 10 days for a reliable quantitative count. The Cryptococcal antigen (CRAG) is a point of care test that rapidly detects cryptococcal polysaccharide capsule using gold-conjugated anti-cryptococcal monoclonal antibodies directed against C. neoformans ${ }^{(7)}$.

Treatment for cryptococcal meningitis is divided into three phases: 1) induction, 2) consolidation, and 3) maintenance phase. Rapid sterilization of cerebrospinal fluid is the focus of induction therapy. Standard treatment is with amphotericin $\mathrm{B}$, flucytosine during the induction phase, and fluconazole during the consolidation and maintenance phase. HAART to be started after a minimum of $2 \mathrm{wks}$ of induction phase in a patient with HIV infection.

\section{Case Description/Summary}

A 37-year-old heterosexual male presented with severe headache, fever, vomiting, and altered sensorium of ten days duration to the local hospital. There he was diagnosed to have pyogenic meningitis and was empirically started on intravenous dexamethasone, ceftriaxone, and vancomycin. While on treatment, he developed bilateral painless loss of vision and bilateral asymmetric lower limb lower motor neuron type weakness with urinary retention, and so he was referred to our hospital for further evaluation and treatment. He had no co-morbidities, habituations, or addictions.

Upon arrival at our institution, he was diagnosed with meningoencephalitis. Clinical examination revealed neck stiffness, positive kernig's and brudzinski's sign, oral candidiasis, seborrhoeic dermatitis, and multiple papulonodular cutaneous lesions. The perception of light was absent in both eyes with a bilateral pale optic disc. The patient was in altered sensorium GCS: E4V1M4, left pupil was sluggishly reactive and positive for swinging flashlight test suggestive of relative apparent pathway defect (RAPD), asymmetric proximal lower limb weakness with diminished deep tendon reflexes and equivocal bilateral plantar response. These findings were suggestive of possible spinal arachnoiditis. Upon further investigation found to have HIV-1 based on reactive HIV ELISA and his CD 4 count was 35 cells $/ \mu 1$.

CSF analysis showed an elevated opening pressure of $440 \mathrm{~mm}$ of $\mathrm{H} 2 \mathrm{o}$, no cells, hypoglycorrhychia, and elevated protein levels. CSF and serum cryptococcal antigen test [CRAG] by Lateral flow assay was positive.CSF India ink staining demonstrated encapsulated budding yeast cells (Fig 2). CSF AFB stain; gene expert MTB/Rif assay and VDRL were negative. MRI brain showed multiple hypointense lesions on T1 and hyperintense lesions on $\mathrm{T} 2$ and FLAIR sequences on the right temporal and left frontal lobes.

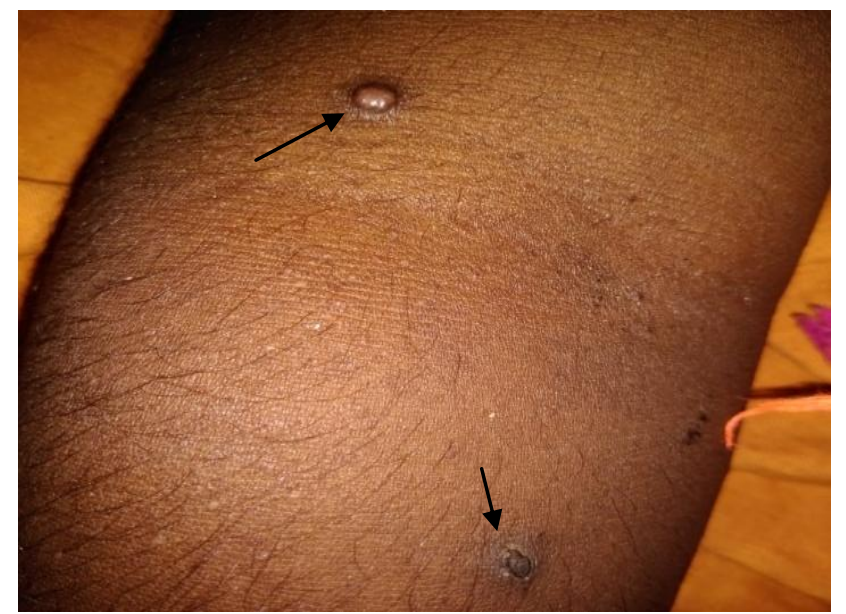

Figure 1: Arrows in this picture depicting papulonodular lesions over forearmCryptococcoma of skin 


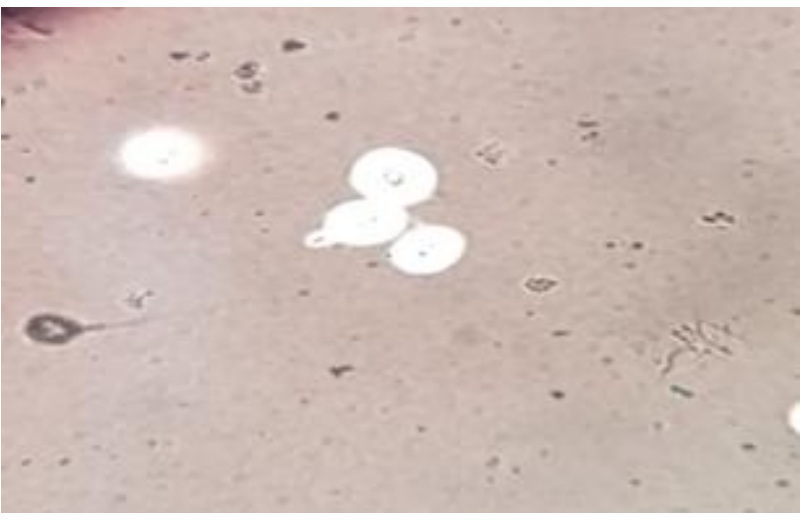

Figure 2: India ink preparations demonstrating encapsulated yeast forms.

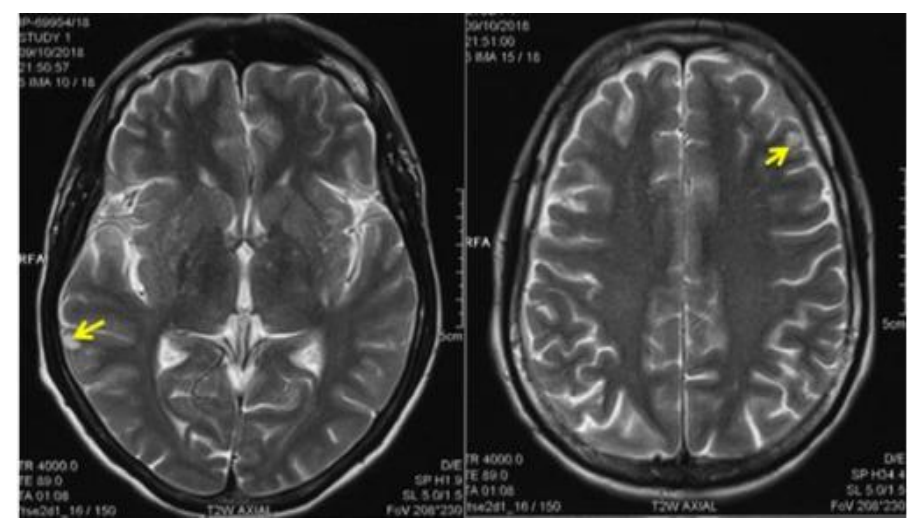

Figure 3: MRI brain $\mathrm{T} 2 \mathrm{w}$ image showing a hyperintense lesion in the right temporal and left frontal lobe suggestive of cryptococcoma in the brain.

Based on the clinical features, biochemical and microbiological results, he was diagnosed to have advanced HIV infection with cryptococcemia, cryptococcal meningoencephalitis, spinal arachnoiditis, multiple cryptococcoma's in the brain with possible cryptococcal infiltration into the optic nerves and tract resulting in binocular visual loss. He was initiated on Amphotericin B deoxycholate $1 \mathrm{mg} / \mathrm{kg} /$ day for one month and flucytosine $100 \mathrm{mg} / \mathrm{kg} / \mathrm{day}$ for 2 weeks. The patient was maintained on $400 \mathrm{mg}$ per day of fluconazole.

Following initiation of specific anticryptococcal therapy, the patient's sensorium improved but there was no perceptible improvement in vision despite completion of the induction phase. Relative afferent pupillary defect made us think about bilateral optic neuritis was present in both eyes and so the patient was started on Intravenous Methylprednisolone $500 \mathrm{mg}$ daily for 5 days followed by Prednisolone $1 \mathrm{mg} / \mathrm{kg}$.MRI of orbit did not reveal thickening or enhancement of optic nerves, tract, or chiasma. Visual evoked potential [VEP] did not pick up any visible waveforms. Spinal arachnoiditis abated after initiation of steroids as evidenced by improvement in lower limb power but improvement in visual acuity has been very gradual with the patient regaining light perception only after 3 weeks of steroids. Antiretroviral therapy with tenofovir, emtricitabine, and efavirenz was started after 6 weeks of initiation of anticryptococcal therapy.

\begin{tabular}{|l|c|c|c|c|}
\hline CSF & At presentation & $2^{\text {nd }}$ week & $3^{\text {rd }} w e e k$ & $4^{\text {th }}$ week \\
\hline CSF PRESSURE & $44 \mathrm{~cm}$ & $36 \mathrm{~cm}$ & $33 \mathrm{~cm}$ & $28 \mathrm{~cm}$ \\
\hline TC & NO CELLS & NO CELLS & 1 & $\begin{array}{c}10 \mathrm{cells} \\
\text { P3L7 }\end{array}$ \\
\hline SUGAR & $27(120)$ & $30(138)$ & $36(126)$ & $71(110)$ \\
\hline PROTEIN & 189 & 145 & 130 & 98 \\
\hline
\end{tabular}

\section{Discussion}

Chronic meningitis must be evaluated and CSF for cryptococcal antigen, Indian ink preparation are must for identification of cryptococcal infection. The lung is the most common mode of entry which on chest $\mathrm{X}$-rays seen as noncalcified single or multiple nodules, indistinct mass-like infiltrates, hilar lymphadenopathy, lobar infiltrates, pleural effusions, and lung cavitation. A lumbar puncture should be considered to rule 
out CNS infection, even in the absence of symptoms in immunocompromised. Acute, subacute, chronic meningitis,cryptococcomas of the brain (abscesses), spinal cord granuloma, chronic dementia (from hydrocephalus). The clinical features of cryptococcal meningitis are headache (80 92\% of cases), meningeal signs (50 80\%), nausea/vomiting (40 80\%), fever (36 67\%), and visual disturbances (33 47\%).

About $50 \%$ of patients have accompanying ocular complications, such as papilledema, cranial nerve palsy, and late-term vision $\operatorname{loss}^{(8)}$. Whereas 7 percent of AIDS patients had cryptococcal infection diagnosed over the course of the illness, only 1.9 percent of cases initially had a cryptococcal infection $^{(9,10)}$.

Comprehensive binocular blindness as the first appearance of HIV-related cryptococcal meningitis is exceedingly $\operatorname{rare}^{(11)}$. Possible mechanisms for binocular blindness due to cryptococcal meningitis include direct fungal invasion of the optic nerve, optic chiasm or optic tract, adhesive arachnoiditis, cerebral vasculitis, and intracranial hypertension. It has been proposed that rapid-onset visual loss is caused by infiltration of the optic nerve or optic chiasm, whereas slow-onset visual loss is caused by increased CSF pressure ${ }^{(12)}$. Neurophthalmic lesions are present in $6 \%$ of patients with HIV infection during the disease, most of which are owing to cryptococcal meningitis ${ }^{(13)}$.

However, the complexities in the neurological evaluation resulting from both the upper and lower motor neuron signs and symptoms can obscure the diagnosis, leading to identification. Relevant to spinal anatomy, lumbar involvement can present with cauda equina or conus medullaris syndromes characterized by bladder dysfunction, lower back and/or leg pain (often bilateral), saddle sensory disturbance, and gait disturbance due to lower extremity weakness and reflex abnormalities, which may be asymmetrical ${ }^{(14)}$. While meningoencephalitis and cryptococcomas are the most common cryptococcal CNS syndromes, spinal arachnoiditis has also been documented in both immunocompromised and previously healthy adults $^{(15,16)}$. Treatment of cerebral cryptococcoma is amphotericin B with flucytosine for at least 6weeks followed by consolidation and maintenance phase for 618 months with fluconazole.Surgery (debulking) if lesion $>3 \mathrm{~cm}$.Corticosteroid if mass effect and edema.Mannitol/acetazolamide is not helpful in cryptococcal meningitis.

\section{Conclusion}

In immunocompromised patients presenting with signs and symptoms conclusive of meningitis, Lumbar puncture should be done and sent for Indian ink preparation, to identify cryptococcii. Since cryptococcal meningitis has high mortality rates. Early antifungal therapy has to be initiated. A thorough search of the literature was done, it is seen that possible mechanisms due to cryptococcal meningitis include direct fungal infiltration into the optic nerve, optic chiasm, and optic tracts, adhesive arachnoiditis, cerebral vasculitis, and intracranial hypertension. Intracranial hypertension causes slow onset and causes progressive vision loss whereas fungal infiltration of the optic nerve, optic chiasm, and optic tracts, cerebral vasculitis, and ischemia causes sudden visual loss ${ }^{(5)}$. HAART therapy has to be initiated once the patient stabilizes with signs and symptoms at least after 2 weeks of antifungal treatment.

\section{References}

1. Powderly WG. Cryptococcal meningitis and AIDS. Clinical infectious diseases. 1993;17(5):837-842.

2. Mirza SA, Phelan M, Rimland D, Graviss E, Hamill R, Brandt ME, et al. The changing epidemiology of cryptococcosis: an update from population-based active surveillance in 2 large metropolitan areas, 1992-2000. Clinical infectious diseases. 2003;36(6):789-794.

3. Colombo AC, Rodrigues ML. Fungal colonization of the brain: anatomopathological aspects of neurological cryptococcosis. Anais da 
Academia Brasileira de Ciências. 2015;87(2):1293-1309.

4. Nosanchuk JD, Casadevall A. The contribution of melanin to microbial pathogenesis. Cellular microbiology. 2003;5(4):203-223.

5. Boulware DR, Rolfes MA, Rajasingham $\mathrm{R}$, von Hohenberg M, Qin Z, Taseera K, et al. Multisite validation of cryptococcal antigen lateral flow assay and quantification by laser thermal contrast. Emerging infectious diseases. 2014;20(1):45.

6. Kisenge PR, Hawkins AT, Maro VP, Mchele JP, Swai NS, Mueller A, et al. Low CD4 count plus coma predicts cryptococcal meningitis in Tanzania. BMC infectious diseases. 2007;7(1):39.

7. Rhein J, Boulware DR. Prognosis and management of cryptococcal meningitis in patients with human immunodeficiency virus infection. Neurobehav HIV Med. 2012;4:45-61.

8. Kestelyn PG, Cunningham Jr ET. HIV/AIDS and blindness. Bulletin of the World Health Organization. 2001;79:208213.

9. Giberson TP, Kalyan-Raman K. Cryptococcal meningitis: Initial presentation of acquired immunodeficiency syndrome. Annals of emergency medicine. 1987;16(7):802.

10. Johnston SRD, Corbett EL, Foster O, Ash $\mathrm{S}$, Cohen J. Raised intracranial pressure and visual complications in AIDS patients with cryptococcal meningitis. Journal of Infection. 1992;24(2):185-189.

11. Hong Y-J, Jung S, Kim J-Y, Kwon S-B, Song K-B, Hwang S-H, et al. Complete Binocular Blindness as the First Manifestation of HIV-Related Cryptococcal Meningitis. J Clin Neurol. 2007 Dec;3(4):212-4.
12. Claus JJ, Portegies P. Reversible blindness in AIDS-related cryptococcal meningitis. Clinical neurology and neurosurgery. 1998;100(1):51-52.

13. Jabs DA. Ocular manifestations of HIV infection. Transactions of the American Ophthalmological Society. 1995;93:623.

14. Panackal AA, Komori M, Kosa P, Khan O, Hammoud DA, Rosen LB, et al. Spinal Arachnoiditis as a Complication of Cryptococcal Meningoencephalitis in NonHIV Previously Healthy Adults. Clinical Infectious Diseases: An Official Publication of the Infectious Diseases Society of America. 2017 Feb 1;64(3):275.

15. Jongwutiwes U, Malathum K, Sungkanuparph S. Cryptococcal meningoradiculitis: an atypical presentation after initiation of antiretroviral therapy. Journal of the Medical Association of Thailand= Chotmaihet thangphaet. 2007;90:85.

16. Stein SC, Corrado ML, Friedlander M, Farmer P. Chronic mycotic meningitis with spinal involvement (arachnoiditis): a report of five cases. Annals of Neurology: Official Journal of the American Neurological Association and the Child Neurology Society. 1982;11(5):519-524. 